\title{
TDOA estimation of dual-satellites interference localization based on blind separation
}

\author{
SU Ting* and GAO Yong \\ College of Electronics and Information Engineering, Sichuan University, Chengdu 610065, China
}

\begin{abstract}
The time difference of arrival (TDOA) estimation plays a crucial role in the accurate localization of the satellite interference source. In the dual-satellites interference source localization system, the target signal from the adjacent satellite is likely to be interfered by the normal communication signal with the same frequency. Therefore, the signal to noise ratio (SNR) of the target signal would become too low, and the TDOA estimation through cross-correlation processing would be unreliable or even unattainable. This paper proposes a technique based on blind separation to solve the co-channel interference problem, where separation of the mixed signal can be carried out by the particle filter (PF) algorithm. The experimental results show that the proposed method could achieve more accurate TDOA estimation. The measured data obtained by using the software radio platform at $915 \mathrm{MHz}$ and $2 \mathrm{GHz}$ respectively verify the effectiveness of the proposed method.
\end{abstract}

Keywords: time difference of arrival (TDOA), co-channel interference, blind separation, particle filter (PF).

DOI: $10.21629 /$ JSEE.2019.04.07

\section{Introduction}

With the rapid development of satellite communication, the interference of terrestrial radiation sources to satellites is increasing, which affects the normal communication of satellites. The problem of satellite interference source localization has become an important content of anti-jamming of satellite communication systems. In the current satellite communication system, satellite interference source localization has become an important part of anti-interference [1]. At present, the successful development of the satellite interference source localization system mainly includes the British SatID system, the US TLS 2000 system and the French HyperLoc system. These systems above are dual-satellites localization methods that use time difference and frequency difference joint positioning,

\footnotetext{
Manuscript received December 28, 2017.

*Corresponding author.

This work was supported by the Fundamental Research Funds for the Central Universities (2082604194194).
}

and the localization accuracy of these systems are in the range of 5-100 km. Thereby parameter estimation has a very important significance in satellite interference localization. Precise extraction of the time difference of arrival (TDOA) plays a key role in accurately locating the source of interference. TDOA estimation has been widely used in interference source localization [2]. In the dual-satellites interference source localization system, dual-satellites localization is carried out by receiving the target signal with the main satellite as well as the target side lobe signal with the adjacent satellite. Notably, it is a very common scenario in which the target signal and the normal communication signal of the adjacent satellite would share the same frequency. In the task of interference source localization, the normal communication signal of the adjacent satellite is regarded as the co-channel interference of the target signal. Therefore, the ground station receives the target signal including the co-channel interference signal from the adjacent satellite, which results in a strong interference to the target signal with a low signal to noise ratio (SNR). Therefore, the TDOA cannot be accurately extracted, which seriously restricts the performance of the interference source localization system. The TDOA could be obtained by cross-correlation processing [3]. Therefore, in order to accurately estimate TDOA, it is necessary to separate the mixed signal forwarded by the adjacent satellite or suppress the co-channel interference signal to obtain the target signal, and then perform correlation processing with the signal forwarded by the main satellite to obtain an accurate TDOA.

There have been few research to investigate the problem that the adjacent satellite has co-channel interference signals in a dual-satellites interference localization system. In [4], the authors proposed a spatial filtering method which could only partially offset the mixed signal. What's more, when the direction of the target signal was nearly consistent with that of the co-channel interference signal, the performance of the spatial filtering method would de- 
grade rapidly. In [5], the independent component analysis (ICA) method was applied to the blind separation of multichannel signals. In [6], the decorrelation filter was used to separate signals in the multi-machine environment. However, both methods above belong to the multi-channel blind separation category, the signal from the adjacent satellite studied in our work has the single-channel blind separation problem of co-channel interference, and the target signal cannot be accurately separated using both of the algorithms above. The two-dimensional search method adopted in [7] can effectively estimate the time delay and the calculation amount was really less, but this method was only suitable for cooperative communication, and not suitable for the time delay estimation of the interference localization problem in non-cooperative communication because it utilized the locally stored auxiliary data. In [8], the estimation of signal parameters via rotational invariance techniques (ESPRIT) algorithm was used to estimate time delay, but it was necessary to set appropriate sampling filter conditions to obtain good estimation performance. In [9], the variable step size (VSS) learning algorithm and the fast convergence algorithm were compared to estimate the time delay, results showed that it was not easy to choose the appropriate VSS method to estimate time delay, and the learning algorithm failed to perform at a low SNR. The algorithm proposed in [9] is not applicable to the TDOA estimation of mixed signals when the signal forwarded by the adjacent satellite has co-channel interference. In [10], researchers studied the effect of the co-channel interference on the time delay estimation of the cross-correlation method, and pointed out that it will seriously affect the accuracy of estimation. Therefore, in order to solve the TDOA estimation problem, the co-channel interference of the adjacent satellite must be suppressed or eliminated first in this paper. In [11], the authors proposed an interference suppression method based on demodulation cancellation to suppress the co-channel interference. A major limitation of this method is that it is only suitable for the situations that SNR of the co-channel interference signal is much larger than the SNR of the target signal. In underdetermined blind separation [12], the situation of single channel with the cochannel interference signal is special. In 2006, the particle filter (PF) algorithm was firstly applied to the single channel blind separation of mixed signals [13]. It has no special requirements for the signal power, frequency offset and other parameters. This algorithm happens to improve the single-channel co-channel interference problem. Therefore, based on blind signal separation techniques, this paper proposes a method to separate the mixed signal and obtain the target signal using the PF algorithm. Remarkably, this method does not require the co-channel interference signal to be much stronger than the target signal. In addition, it can track parameters dynamically and is adaptable. The accurate TDOA can be obtained by the cross-correlation processing of the separated target signal and the signal from the main satellite. Under the carrier frequencies of $915 \mathrm{MHz}$ and $2 \mathrm{GHz}$ separately, the effectiveness of the proposed method has been validated by our experimental results with the real measured data received from the NI USRP-2930 software radio platform.

\section{Principle of localization}

In a dual-satellite localization system, the main beam of interference source usually points to the main satellite and the side beam points to the adjacent satellite due to the characteristics of the emitter antenna of the interference source. Signals transmitted from the target interference source emitter are transmitted by two different satellites, and the transmitted signals are received by two different ground stations in the satellite covered areas. In this case, two different satellites transmit the same source signals' main beam and side beam from the target interference source emitter, which would lead to different propagation paths and a time difference. If the location of the dualsatellite and the receiving station is known, time difference contour line on the ground can be established, which extends the ground longitude direction to form a hyperboloid and intersects a curve on the ground. In this way, the target interference source emitter can be desirably localized on this hyperbola. However, there often exists the normal communication signal, and its frequency is the same with the target signal on the adjacent satellite, so it is considered as the co-channel interference signal of the target signal. Therefore, in the presence of the co-channel interference, the principle of the dual-satellites localization system can be shown in Fig. 1.

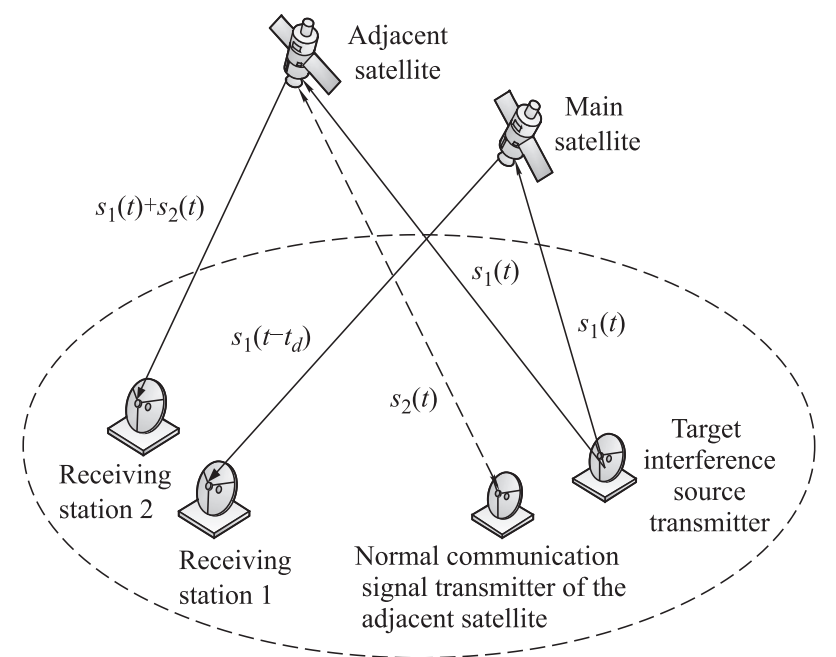

Fig. 1 Principle diagram of the dual-satellite localization 
In Fig. $1 s_{1}(t)$ represents the target signal transmitted by the target interferer transmitter, and $s_{2}(t)$ denotes the co-channel interference signal forwarded by the adjacent satellite normal communication signal transmitter, and $t_{d}$ is the time difference between the arrival of the two different ground receivers.

\section{Algorithm research}

\subsection{Signal model}

The signal model can be formulated as follows:

$$
\left\{\begin{array}{l}
x(t)=s_{1}\left(t-t_{d}\right)+n_{1}(t) \\
y(t)=u \times s_{1}(t)+s_{2}(t)+n_{2}(t)
\end{array}\right.
$$

where $x(t)$ denotes the signal from the main satellite and $y(t)$ denotes the mixed signal from the adjacent satellite. The target signal and the interference signal are denoted by $s_{1}(t)$ and $s_{2}(t)$ respectively. Moreover, $t_{d}$ represents the time difference between the target signals and the two ground receivers and $u$ is the attenuation coefficient. Finally, $n_{1}(t)$ and $n_{2}(t)$ are additive white Gaussian noise (AWGN), which are independent of the target signal and the interference signal.

In general, considering a complex baseband model, the target signal and the interference signal can be expressed as

$$
s_{i}(t)=h_{i} \mathrm{e}^{\mathrm{j}\left(\Delta \omega_{i} t+\theta_{i}\right)} \sum_{n} a_{i, n} g_{i}(t-n T), \quad i=1,2
$$

where the amplitude of two signals is denoted by $h_{i}$, the residual carrier is denoted by $\Delta \omega_{i}$, the initial phase is denoted by $\theta_{i}$, and the information code is denoted by $a_{i, n}$. The equivalent channel filter is represented by $g_{i}(t)$, which is usually assumed to be a raised cosine filter due to the ideality of the satellite communication channel. The symbol period is denoted as $T$, and the duration of the raised cosine filter is then assumed to be $L T$. In particular, the duration of the raised cosine filter is within the interval $\left[\left(1+L_{1}\right) T, L_{2} T\right], L=L_{1}+L_{2}$.

In this paper, the target signal and the interference signal are both binary phase shift keying (BPSK) modulated. Therefore, we have

$$
a_{i, n}=\mathrm{e}^{\mathrm{j} 2 \pi l / M}, \quad i=1,2 ; l=0,1, \ldots, M-1 ; M=2 .
$$

By sampling the received signal with the symbol rate, we can get

$s_{i, k}=h_{i, k} \mathrm{e}^{\mathrm{j}\left(\Delta \omega_{i} k T+\theta_{i}\right)} \sum_{n} a_{i, n} g_{i}(k T-n T), \quad i=1,2$.
By introducing the $L \times 1$ vector, it can be written

$$
\begin{gathered}
\overline{\boldsymbol{g}}_{i, k}=h_{i, k} \mathrm{e}^{\mathrm{j}\left(\Delta \omega_{i} k T+\theta_{i}\right)}\left[g_{i}\left(L_{1}-1\right) T,\right. \\
\left.g_{i}\left(L_{1}-2\right) T, \ldots, g_{i}\left(-L_{2} T\right)\right]^{\mathrm{T}} \cdot \\
\overline{\boldsymbol{a}}_{i, k}=\left[a_{i, k-L_{1}+1}, a_{i, k-L_{1}+2}, \ldots, a_{i, k+L_{2}}\right]^{\mathrm{T}} .
\end{gathered}
$$

Therefore, the mixed signal received from the adjacent satellite can be given by

$$
y_{k}=\overline{\boldsymbol{g}}_{i, k} \overline{\boldsymbol{a}}_{i, k}+n_{2, k} .
$$

\subsection{PF}

The procedures of blind separation based on the PF method can be summarized as follows [14].

Step 1 Initialization: sample with initial distribution and obtain $N$ initial particle points $x_{0}^{(i)}(i=1,2, \ldots, N)$.

Step 2 Importance sampling: from time $k-1$ to time $k$, assume that the state of the particle at time $k$ is $x_{k-1}^{(i)}$, and the corresponding importance weight is $w_{k-1}^{(i)}$, for each particle $i=1,2, \ldots, N$, obtain new particles by the importance sampling function.

Step 3 Weight update: update the particle weights $w_{k}^{(i)}$ by the updating formula of importance weights and normalize the weights by $\widetilde{w}_{k}^{(i)}=w_{k}^{(i)} / \sum_{i=1}^{N} w_{k}^{(i)}$.

Step 4 Resample: set $N_{t h}$ as a threshold, calculate "effective particles" $N_{\text {eff }}$ by formula $N_{\text {eff }} \approx$ $1 / \sum_{i=1}^{N}\left(w_{k}^{(i)}\right)^{2}$. Resample if $N_{e f f}$ is less than the threshold $N_{t h}$ and re-initialize importance weights of all particles to $1 / N$.

Step 5 Symbol estimate: estimate symbols by (7), return to Step 2 and continue the whole iterative process until all symbols are estimated.

\subsection{TDOA estimation}

The flow chart of TDOA estimation is shown in Fig. 2. Once the symbol sequence has been obtained from the blind source separation procedure, the target signal can be got by modulation again in the ground station, then the TDOA can be calculated by cross-correlation with the target signal and the signal from the main satellite. For the signal modulation, the amplitude, initial phase and frequency offset of the carrier are all required to be known. The amplitude and initial phase can be estimated by using the PF algorithm in the blind source separation step. Frequency offset can be estimated by the non-data aided carrier frequency offset estimation algorithm [15]. 


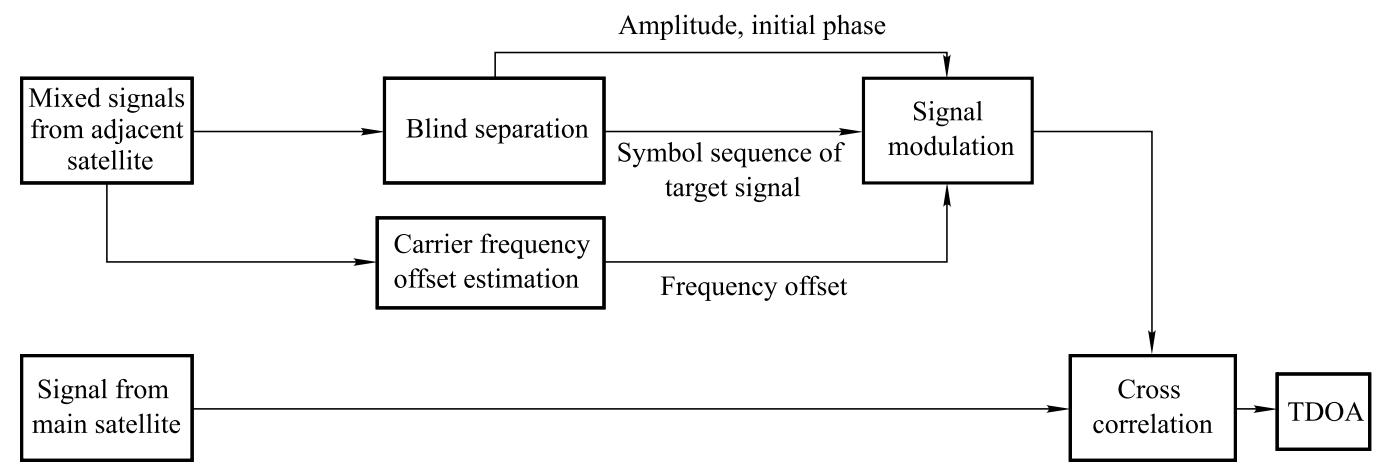

Fig. 2 Flow diagram of TDOA estimation

\section{Experiments and results}

Simulation experiments are carried out to validate the proposed algorithm. In these simulations, two BPSK modulated signals with a symbol rate of $2.5 \mathrm{Mbps}$ and a sampling rate of $20 \mathrm{MHz}$ are used as the target signal and the co-channel interference signal separately. Raised cosine filters with roll-off factor 0.35 and $L=4$ are used as the equivalent channel filters. The number of particles is chosen to be 300 .

In order to fairly evaluate the performance of TDOA estimation before and after separation, 100 Monte-Carlo trials separately based on different data lengths are carried out. The statistical results show that the error of TDOA estimation changes with the data length. The simulation results are shown in Fig. 3.

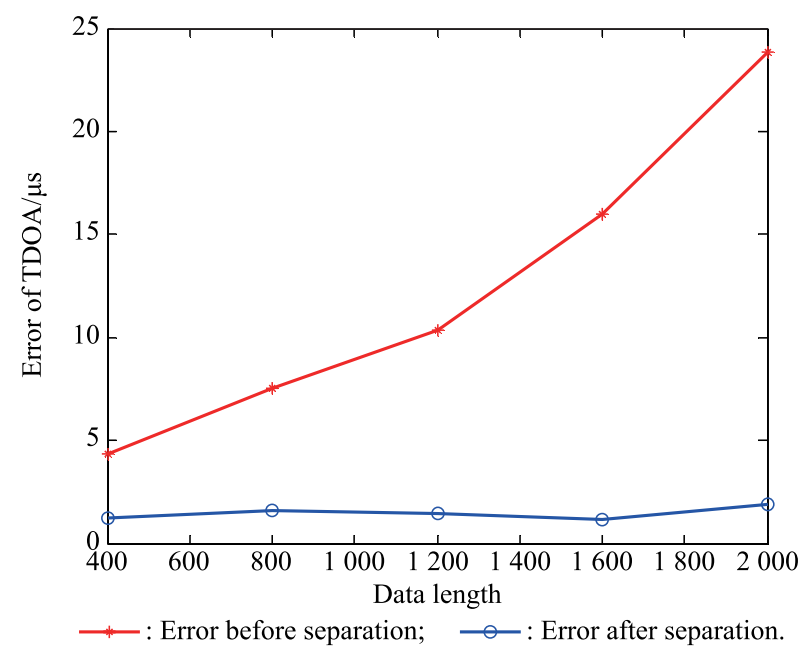

Fig. 3 Error of TDOA estimation before separation and after separation

It can be clearly seen from Fig. 3 that the TDOA obtained by blindly separating the mixed signals of the adjacent satellite and then correlating with the signal of the main satellite is significantly more accurate than the TDOA obtained without separation. Since the preset time delay value is fixed, under the condition of a fixed sampling period, longer data could induce larger corresponding time range. However, due to the presence of the co-channel interference signal, longer data before separation could induce greater estimated delay spread range and greater error between the obtained time delay and the real time delay. Since the blind separation process suppresses the cochannel interference signal to obtain a more accurate time delay, even if the data length increases, the error does not change much, and is still at a small value, indicating the method proposed in this paper is effective in terms of different data lengths.

Moreover, in order to illustrate the advantage of the proposed method, the length of data is chosen to be 2000 , the SNR is set to be $-5 \mathrm{~dB}$, the amplitude of the target signal is 0.4 , the amplitude of the co-channel interference signal is 1 , and time delay $t_{d}$ between the signal from the main satellite and that from the adjacent satellite is $30 \mu \mathrm{s}$. Firstly, the mixed signal is directly used to estimate the time delay. Then, before separation, the third-order cumulant and fourth-order cumulant algorithms are used to estimate the time delay respectively. And then the ICA and the proposed PF method are used to separate the mixed signal separately. Finally, the time delay estimation can be obtained by the cross-correlation processing of the separated target signal and the signal from the main satellite. The simulation results are shown in Fig. 4 -Fig. 8.

As shown in Fig. 4-Fig. 6, the peak cannot be directly detected if the mixed signal from the adjacent satellite is not separated, so the TDOA is not obtained. Therefore, without special pre-processing, existing delay estimation methods are not applicable at all in the case of our study. In Fig. 7 and Fig. 8, the experimental TDOA estimation results are obtained by using the ICA method and the proposed PF method to separate the mixed signal and then correlating with the main satellite signal respectively. The peak cannot be detected, so the TDOA cannot be accurately estimated even if the ICA method is used. By us- 
ing the PF algorithm to separate the mixed signal, the co-channel interference signal can be substantially suppressed, and the SNR of the target signal from the adjacent satellite can be effectively improved. As a result, more accurate TDOA can be obtained.

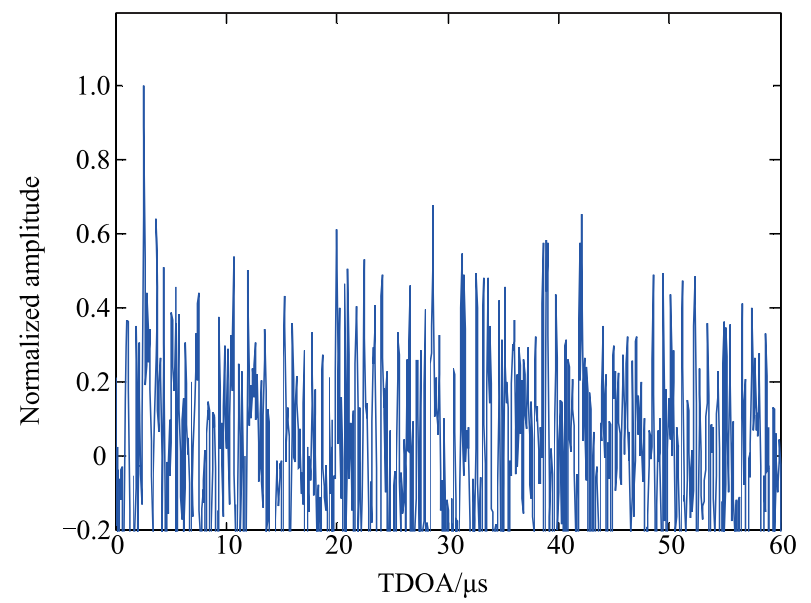

Fig. 4 TDOA based on cross-correlation

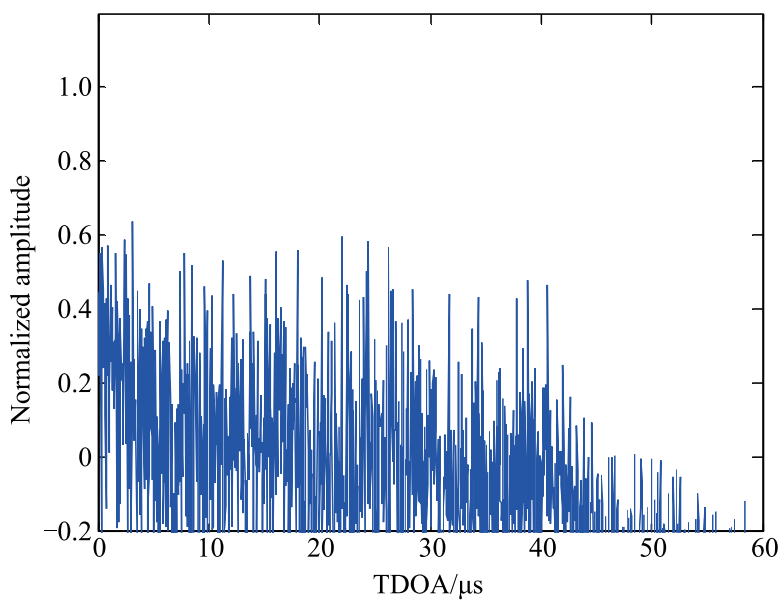

Fig. 5 TDOA based on third-order cumulant

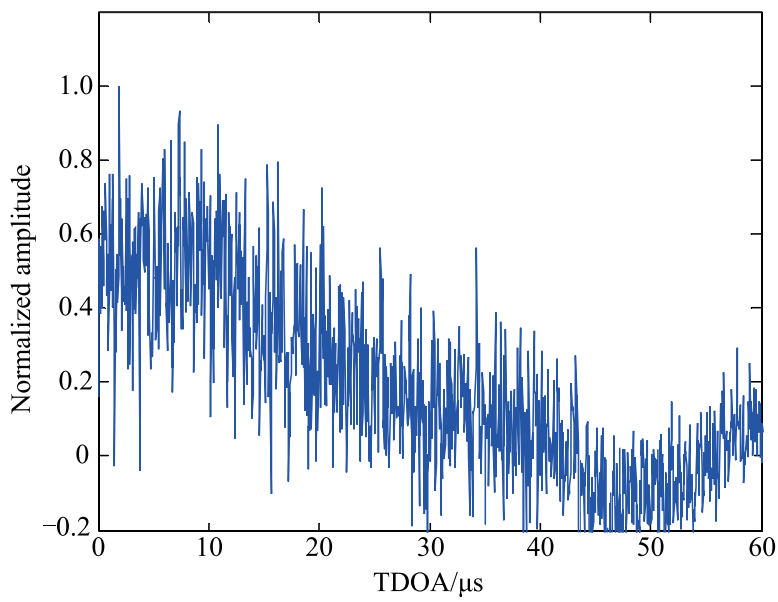

Fig. 6 TDOA based on fourth-order cumulant

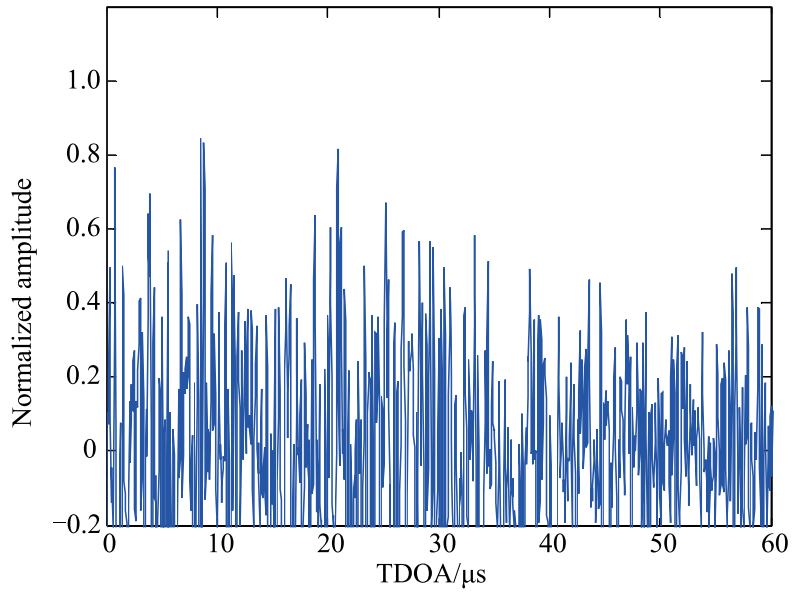

Fig. 7 TDOA based on ICA

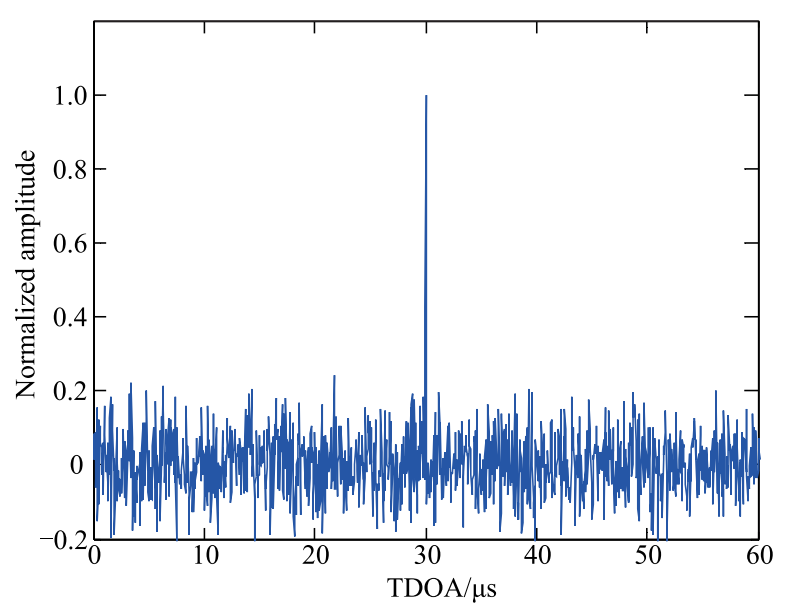

Fig. 8 TDOA based on PF

Using the ICA algorithm and the PF algorithm to separate the mixed signals under different SNR conditions respectively, and then using the cross-correlation method to estimate the time delay, the simulation results are shown in Table 1.

Table 1 TDOA based on ICA and PF under different SNRs

\begin{tabular}{ccc}
\hline \multirow{2}{*}{$\mathrm{SNR} / \mathrm{dB}$} & \multicolumn{2}{c}{$\mathrm{TDOA} / \mu \mathrm{s}$} \\
\cline { 2 - 3 } & $\mathrm{ICA}$ & $\mathrm{PF}$ \\
\hline 0 & 12.25 & 30 \\
3 & 21.80 & 30 \\
5 & 1.25 & 30 \\
\hline
\end{tabular}

Table 1 indicates that the accurate TDOA based on the ICA algorithm is not obtained, while the accurate TDOA based on the PF algorithm can be obtained.

In order to validate the effectiveness of the proposed method, experiments with real measured data are carried out. In particular, the NI USRP-2930 software radio platform is chosen to transmit the BPSK modulation signal 
with a carrier frequency of $915 \mathrm{MHz}$. The number of symbols is 4000 , the symbol rate is $1.25 \mathrm{Mbps}$, the sampling rate is $10 \mathrm{MHz}$, the roll-off factor of the matched filter is 0.35 , and the filter length is 4 . The signal is received through the software radio platform, then the measured data are firstly separated using the proposed PF method, and the TDOA is obtained by the cross-correlation processing. The results of time delay estimation before separation and after separation are presented in Fig. 9.

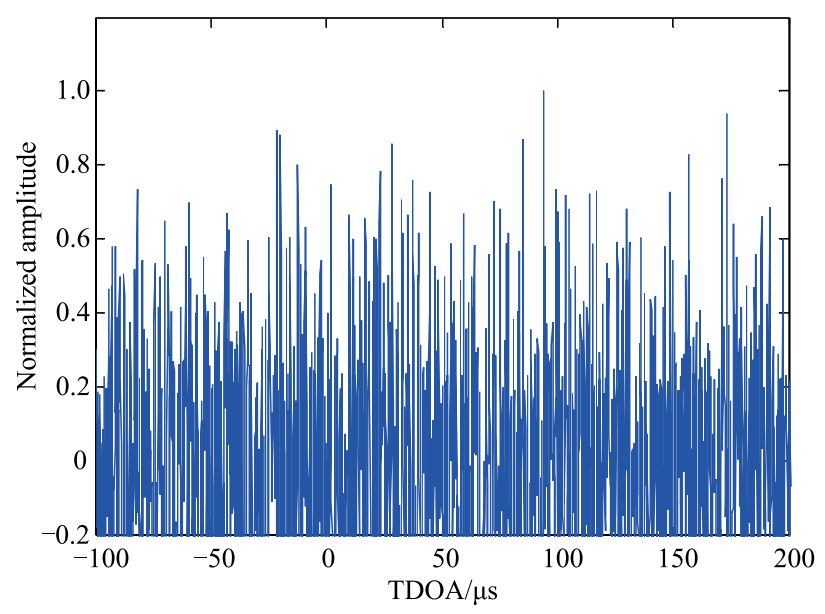

(a) TDOA based on cross-correlation

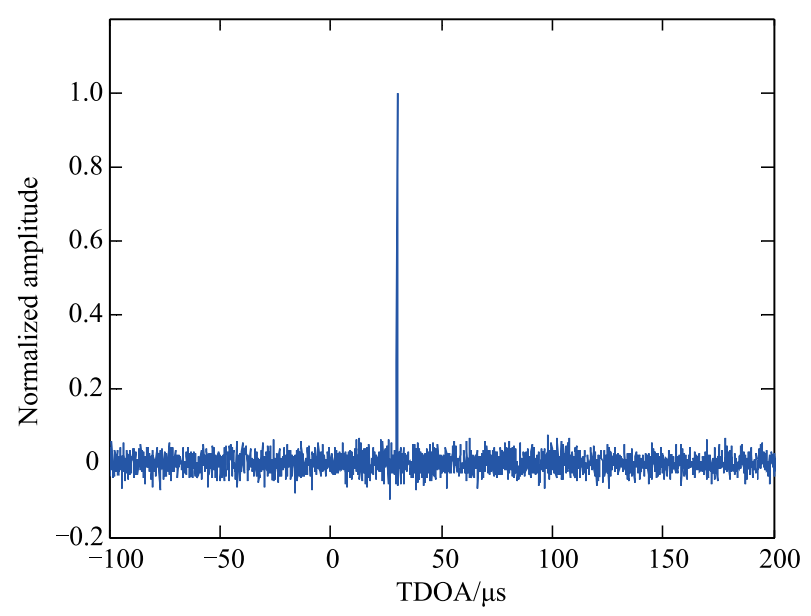

(b) TDOA based on PF

Fig. 9 Experimental results with carrier frequency of $915 \mathrm{MHz}$

In the mentioned experiments above, when carrier frequency is changed to $2 \mathrm{GHz}$ and other parameters remain invariable, the experimental results are given in Fig. 10.

As shown in Fig. 9 and Fig. 10, the TDOA can be obtained at the carrier frequencies of $915 \mathrm{MHz}$ and $2 \mathrm{GHz}$ separately.

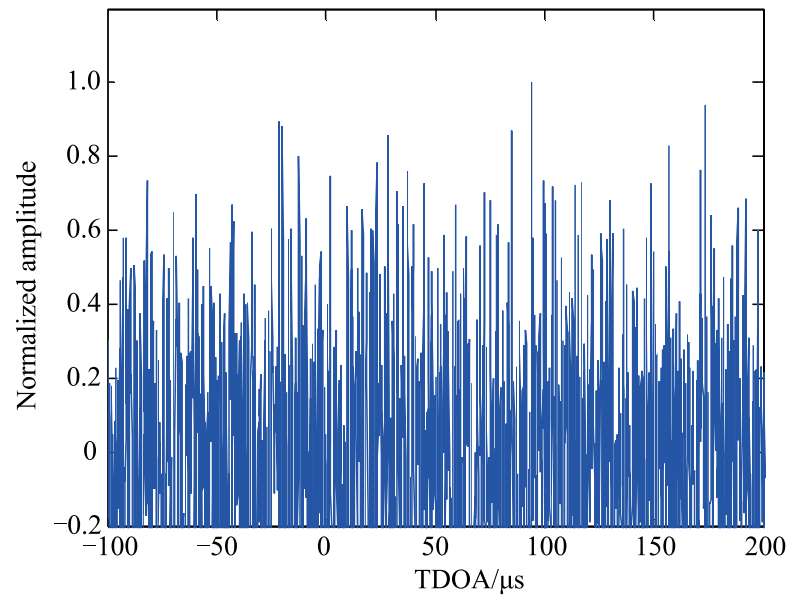

(a) TDOA directly based on cross-correlation

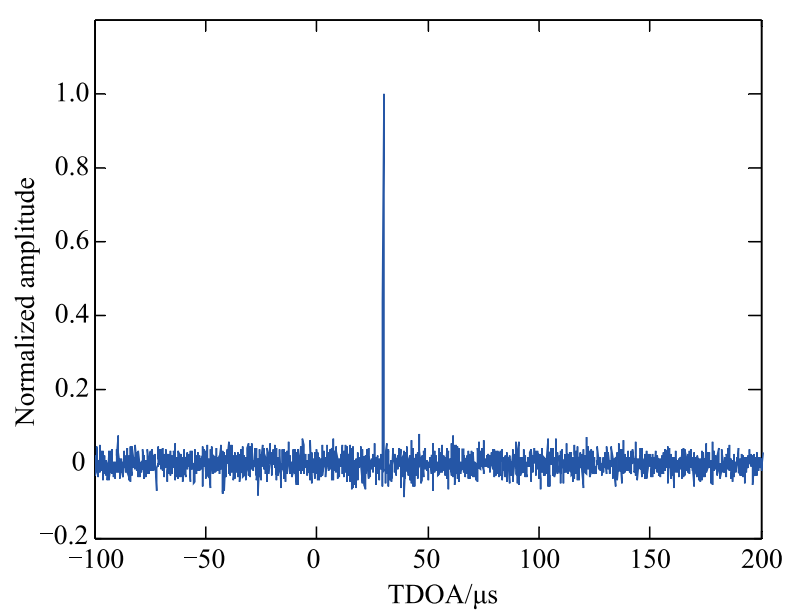

(b) TDOA based on PF

Fig. 10 Experimental results with carrier frequency of $2 \mathrm{GHz}$

\section{Conclusions}

In this paper, a blind signal separation technique is applied to solving the problem of co-channel interference. For the TDOA estimation in dual-satellites interference localization systems, this method can effectively suppress co-channel interference from adjacent satellites, and can desirably improve TDOA estimation accuracy. The proposed method is tested with practically measured data that are received by the software radio platform at the frequencies of $915 \mathrm{MHz}$ and $2 \mathrm{GHz}$ respectively. The effectiveness of the proposed method is demonstrated by the experimental results.

\section{References}

[1] MATSUMOTO Y, TANAKA M, OKAZAWA H. Satellite interference location system using on-board multibeam antenna. Electronics \& Communications in Japan, 1997, 80(11): $22-$ 33.

[2] WEN F, WAN Q, LUO L Y. Time-difference-of-arrival estimation for noncircular signals using information theory. 
AEU-International Journal of Electronics and Communications, 2013, 67(3): 242-245.

[3] WENDEBERG J, SCHINDELHAUER C. Polynomial-time approximation algorithms for anchor-free TDOA localization. Theoretical Computer Science, 2014, 553: 27-36.

[4] YAN K K, LU Y. Sidelobe reduction in array-pattern synthesis using genetic algorithm. IEEE Trans. on Antennas \& Propagation, 1997, 45(7): 1117 - 1122 .

[5] YANG S M. The theory and application of blind source separation ICA. Chengdu, China: University of Electronic Science and Technology, 2009.

[6] BRANDT-PEARCE M. Signal separation using fractional sampling in multiuser communications. IEEE Trans. on Communications, 2002, 48(2): 242-251.

[7] WEI X J, YANG Y, GUO Y M, et al. Joint estimation of interference frequency offset and time delay in PCMA system. Journal on Communication, 2017, 38(2): 173-182.

[8] GEDALYAHU K, ELDAR Y C. Time-delay estimation from low-rate samples: a union of subspaces approach. IEEE Trans. on Signal Processing, 2010, 58(6): 3017-3031.

[9] DOOLEY S R, NANDI A K. Subsample time delay estimation with variable step size control. Signal Processing, 2000, 80(2): $343-347$.

[10] LIANG L T, HAN F, FAN X, et al. Study of effect of coherent interference on time delay estimation. Journal of Detection \& Control, 2007, 29(6): 11-14.

[11] YANG Y X, XIA C X, XIONG J Y. Adjacent satellite cochannel interference cancellation based on demodulation in dual-satellite location system. Telecommunication Engineering, 2012, 52(11): 1730-1735.

[12] LI Y, AMARI S, CICHOCKI A. Underdetermined blind source separation based on sparse representation. IEEE Trans. on Signal Processing, 2006, 54(2): 423-437.
[13] LIU K, LI H, DAI X. Single channel blind separation of cofrequency MPSK signals. Proc. of the 5th IASTED International Conference on Communications, Internet, and Information Technology, 2006: $42-46$.

[14] WAN J, TU S L. Theory and technology on blind source separation of communication signals. Beijing: National Defense Industry Press, 2012.

[15] OSWALD E. NDA based feedforward sampling frequency synchronization for OFDM systems. Proc. of the 59th IEEE Vehicular Technology Conference, 2004: 1068-1072.

\section{Biographies}

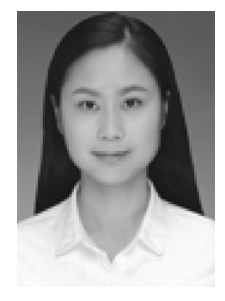

SU Ting was born in 1992. She is a master in Sichuan University. Her research areas mainly include satellite communications and blind signal processing.

E-mail: 929215328@qq.com

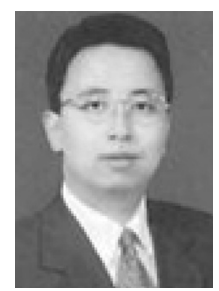

GAO Yong was born in 1969. He is a Ph.D. and a professor in Sichuan University. His research areas mainly include speech signal processing, anti-interference and anti-interception technology in communication, array signal processing, and blind analysis of signal and emergency communication.

E-mail: gaoyong@scu.edu.cn 